\title{
La práctica laboral y su incidencia en la formación inicial de la competencia registrar hechos económicos.
}

DOI: https://doi.org/10.33262/ap.v3i2.1.52

The labor practice and its incidence in the initial formation of the competence to register economic facts.

Elizabet Prado Chaviano. ${ }^{1}$, Margarita González González. ${ }^{2}$, Bisleivys Jiménez Valero. ${ }^{3}$, Ilvia Montalvo Palacios. ${ }^{4}$ \& Luis Efraín Velastegui López. ${ }^{5}$

\begin{abstract}
The labor practice Accountant and Financial in the accounting run and finance occupies a fundamental role in the integral formation of the educandoses of that science, the tasks that it is established in are guide by them this practical contribute in the competitive formation professional and specifically in the initial formation of the competence to register economics facts integrated knowledge's, skills, values, motivations and acting's in the labor activities, it who pays in the presentation of the economics financial information of the enterprise to secure the reliable results in the auditory thing for which the objectives of the investigation is to elaborated a sprouts for practice it labor financial accountant I.
\end{abstract}

Keys word. competence to register economics facts, practice it labor financial accountant

\section{Resumen}

La Práctica Laboral Contable y Financiera en la carrera Contabilidad y Finanzas, ocupa un rol fundamental en la formación integral de los educandos de esa ciencia, las tareas

\footnotetext{
${ }^{1}$ Profesora de la carrera Contabilidad y Finanzas. Universidad de Matanzas. Cuba. Correo electrónico: elizabetpradochaviano7979@gmail.com, Orcid. http//://orcid.org/0000-0002-8101-0440

${ }^{2}$ Profesora investigadora del Centro de Estudios Educativos. Universidad de Matanzas. Cuba. Correo electrónico: gonzalezgonzalez.margarita4@gmail.com, Orcid. http//://orcid.org/0000-0002-3041-6387.

${ }^{3}$ Profesora de la carrera Turismo. Universidad de Matanzas. Cuba. Correo bisleivys.jimenez@umcc.cu Orcid. http//://orcid.org/0000--0003-4812-4558

${ }^{4}$ Profesora de la carrera Contabilidad y Finanzas. Universidad de Matanzas. Cuba. Correo electrónico: ilvia.montalvo@umcc.cu, ORCID: http://orcid.org/ 0000-0002-1938-7001

${ }^{5}$ Ciencia Digital Editorial, Ecuador, luisefrainvelastegui @ cienciadigital.org
} 
que se establecen en las guías de dichas prácticas, contribuyen en la formación de competencias profesionales y específicamente en la formación inicial de la competencia registrar hechos económicos integrando conocimientos, habilidades, valores, motivaciones y desempeños en la entidades laborales, lo cual tributa en la presentación de la información económica financiera de manera veraz y oportuna de las empresas para asegurar resultados confiables en las auditorias por lo que el objetivo de esa investigación es elaborar una guía para la Práctica Laboral Contable y Financiera I.

Palabras claves. Registrar hechos económicos, Práctica Laboral Contable y Financiera.

\section{Introducción}

En las condiciones actuales, resulta de vital importancia para el profesional de las Ciencias Contables y Financieras la formación por competencias de su campo profesional. En ese sentido la Práctica Laboral Contable y Financiera I, permite a los educandos la adquisición en esas competencias a través del estudio e investigación de las técnicas más avanzadas y contribuyen al perfeccionamiento de la gestión económica financiera en las entidades cubanas (Valera, 2009).

La Práctica Laboral Contable y Financiera I, combina de forma articulada los campos de acción del profesional y la integración de conocimientos a nivel de cada año académico de forma vertical en el mapa curricular y de forma articulada a nivel de disciplinas horizontalmente y tiene dentro de sus premisas fundamentales registrar, presentar y analizar la información económica, en entidades productivas y de servicios, aplicando las técnicas, métodos y procedimientos que caracterizan la práctica contable cubana, en correspondencia con las Normas Cubanas de Contabilidad (MES, 2017).

El estudio que se presenta, tiene como objetivo elaborar una guía de la Práctica Laboral Contable y Financiera I, con las tareas a acometer para la formación delos estudiantes, con énfasis en la competencia profesional específica registrar hechos económicos y toma en consideración los modos de actuación, campos de acción y objetivos de la carrera, a través del registro contable del uso de los recursos materiales y financieros de las entidades, en correspondencia con las Normas Cubanas de Contabilidad y las Normas Internacionales de la Información Financiera así como las técnicas que rigen el proceso contable y su presencia dentro de los objetivos generales de la disciplina Contabilidad y de la disciplina integradora Práctica Laboral Contable y Financiera I, en aras de contribuir a la formación de un profesional capaz de registrar, presentar y analizar diferentes hechos económicos vinculados a la práctica contable (MES, 2017), lo cual contribuye a la presentación de la información económica financiera de manera veraz y la toma de decisiones oportunas así como el desempeño demostrado en el puesto de trabajo.

\section{Desarrollo}

La Práctica Laboral Contable y Financiera I, se vincula a las exigencias del Plan de Estudio E, en relación a los modos de actuación, campos de acción y objetivos de la 
carrera, a través del registro contable del uso de los recursos materiales y financieros de las entidades, en correspondencia con las Normas Cubanas de Contabilidad y las Normas Internacionales de la Información Financiera así como las técnicas que rigen el proceso contable y contribuye a la presentación de la información económica financiera de manera veraz.(Prado, 2021). A través de esta disciplina, el estudiante adquiere y desarrolla un carácter científico investigativo, que se forma en la medida que sea promotor activo de su formación, esto se manifiesta en la flexibilidad de gestión que posee la disciplina, al ser instrumento de innovación de los estudiantes y apoyar su formación científica y propicia la formación inicial de la competencia profesional específica registrar hechos económicos (Valera, 2016).

Esa formación inicial integra los sistemas de conocimientos relacionados con la normativa contable, la documentación mercantil, las cuentas y subcuentas como instrumentos de representación y medida de un elemento del patrimonio o de los resultados, contabilizar los hechos económicos en el libro diario, partir de asientos contables y el conocimiento de los procedimientos contables. Asimismo, deben manifestarse los valores éticos del contador tales como: la honestidad, profesionalidad y fidelidad y la colaboración mediante el trabajo en equipo con los especialistas en el área contable en el entorno laboral (Prado, 2018 a y b) así como las relaciones interpersonales y el desempeño alcanzado como modos de actuación profesional (Prado, 2021).

La Práctica Laboral, es una de las vías principales para fomentar y estrechar las relaciones entre la universidad y las empresas, pues contribuye a la formación de competencias en las esferas de actuación del contador, como requisito indispensable para lograr un graduado capaz de analizar, interpretar y dar solución a las distintas situaciones que se deriven de un problema resolver o una situación novedosa que requiera distintas alternativas (Valera, 2009 y Hernández, 2014).

En la formación del estudiante universitario de Contabilidad y Finanzas, en la medida en que se acerca a la vida profesional, esencialmente a través de principios de carácter laboral, logra palpar con más fidelidad la formación académica que cada año de la carrera le ha aportado, al mismo tiempo en que se convierte en investigador de su ciencia, vinculado a los problemas de su profesión (Prado y López, 2020 y Prado y Baujín, 2020).

En el periodo de dicha práctica, se deben desarrollar tareas como el control de las cuentas contables en función de cotejar los importes de las partidas con los físicos en términos de activos, la documentación mercantil con los comprobantes de operaciones y por ende con los saldos correspondientes en los estados financieros, así como la aplicación de procedimientos contables y los modelos, resoluciones, las cuentas y subcuentas y los asientos tipos utilizados en las entidades (Prado, 2017).

Consecuentemente debe contemplarse que en dicha práctica, la integración de contenidos relacionados con las instituciones, las Inversiones y Activos Financieros a Corto y Largo Plazo, el registro y acumulación del costo, los software contables, la estructura del presupuesto del Estado en Cuba, los modelos de regresión evidenciados en el proceso de 
enseñanza aprendizaje de las asignaturas Contabilidad de Gestión I, Matemática, Econometría, Investigación de Operaciones, Finanzas Gubernamentales, Sistema Financiero y Teoría Sociopolítica 'y además se establecen tareas en diferentes departamentos de las entidades y el intercambio con los equipos de trabajo, donde se fomentan los valores éticos del contador, tales como: la honestidad, profesionalidad y fidelidad (Prado, González y Montalvo, 2021).

\section{Metodología}

Para la elaboración de la guía de la Práctica Laboral Contable y Financiera I, se realizó un diagnóstico como parte de un estudio precedente, sustentado en la utilización de un conjunto de métodos que a continuación se detallan:

Tormenta de ideas: se realizó con 10 profesores de la carrera Contabilidad y Finanzas, con el fin de realizar una crítica de las guías de prácticas, mostrando cómo resultado que el 100\% de los profesores señalan que las respuestas de las tareas establecidas en la guía son mecánicas y que existe desconocimiento de la utilización del software contable

El 80\% expresa que las guías son generales, o sea, es la misma para todo tipo de empresa y existe un desnivel en cuanto a las asignaturas del año, se manifiestan unas más que otras mientras el $40 \%$ refiere que no se hace alusión de forma explícita a la vinculación de los contenidos estudiados en clase con el trabajo que realizan en la empresa.

Entrevista: es utilizada para obtener los criterios de los 10 profesores y 65 estudiantes del segundo año del curso diurno. En este caso en su totalidad manifiestan que las prácticas son de gran importancia ya que este es al escenario, donde se consolidan los conocimientos y habilidades adquiridos en clases y contribuye a la preparación del estudiante como egresado de la carrera y refieren la importancia que tiene el vínculo teoría-práctica como una manera más dinámica de ejecutar lo estudiado y despertar el interés por parte de los alumnos, haciendo énfasis en aspectos como la ubicación, que se considera sin dudas primordial para el perfecto desarrollo de esta actividad teniendo en cuenta que el estudiante en este medio, completa su formación profesional.

El $60 \%$ de los profesores y el $69.23 \%$ de los estudiantes plantean que existe una retroalimentación entre estos dos actores, lo cual facilita la organización, planificación, ejecución, control de las actividades y la resolución de las guías, interviniendo en este periodo tanto los profesores de las disciplinas como del colectivo del año.

El $40 \%$ de los profesores reconocen que no existe sistematicidad en las actividades metodológicas para el perfeccionamiento de las guías de prácticas y el $80 \%$ de los estudiantes muestra insatisfacción porque en la mayoría de los casos los trabajadores no están informados de la atención que deben prestar a los alumnos y en disímiles ocasiones no cuentan con el tiempo necesario para ello. A su vez manifiestan los estudiantes, que son muy extensivas las guías y el vínculo que mantienen sus preguntas con las actividades que se realizan en la empresa son factores que critican. 
El $69.23 \%$ de los estudiantes plantean que el vocabulario técnico utilizados por los profesionales en las empresas, no coincide con el de los practicantes y esto dificulta el flujo de información.

Encuesta: aplicada a los tutores que atienden en el período de la Práctica Laboral Contable y Financiera I a los estudiantes de segundo año en las entidades del territorio, donde el $100 \%$ refiere que los estudiantes no saben trabajar con software y no consultan los manuales de normas y procedimientos contables, aunque consideran, que la mayoría de los estudiantes realizan tareas relacionadas con la actualización de los archivos contables e identifican las cuentas con dificultades, pero responden positivamente a las tareas asignadas por la entidad laboral, a su vez, consideran que demuestran valores éticos del contador como la honestidad, responsabilidad y motivación por aprender a registrar los hechos económicos.

Encuesta: para identificar los contenidos principales correspondientes a los objetivos del año se realiza una tabla con los objetivos de cada asignatura. En este caso, las asignaturas que más se trabajaron fueron Contabilidad General III y IV, Estadística Matemática, Sistema Financiero, Teoría Sociopolítica, Informática para la gestión, Investigación de Operaciones, Finanzas Gubernamentales por su repercusión para resolver problemas en las empresas y se dedicó especial atención al registro de los hechos económicos, por su importancia tanto en el proceso de enseñanza como base para las demás disciplinas del perfil profesional, como su repercusión desde el desempeño profesional en los puestos de trabajo de los departamentos económicos de las empresas, en función de la exactitud, trasparencia y confiabilidad de los datos económicos financieros.

A continuación se presenta la guía como sigue:

Universidad de Matanzas

Facultad de Ciencias Empresariales

Departamento de Contabilidad y Finanzas

Guía de Práctica Laboral Contable y Financiera I

Modalidad presencial

Año 2do

Objetivos generales:

1. Aplicar técnicas de valuación, registro y presentación de las partidas fundamentales que integran el activo fijo del Estado de Situación, así como las fuentes de financiamiento a corto y largo plazo, teniendo siempre en cuenta el cumplimiento del marco conceptual de la Contabilidad y os procedimientos contables.

Tareas a desarrollar: 


\begin{tabular}{|c|c|c|c|}
\hline Asignaturas & Objetivos & Preguntas o tareas & Ubicación \\
\hline $\begin{array}{l}\text { Contabilidad } \\
\text { de } \\
\text { Gestión I }\end{array}$ & $\begin{array}{l}\text { 1. Dominar el } \\
\text { proceso de registro y } \\
\text { acumulación de los } \\
\text { costos. }\end{array}$ & $\begin{array}{l}\text { 1. ¿Cómo se lleva el } \\
\text { análisis del costo al } \\
\text { consejo de dirección de la } \\
\text { entidad? } \\
\text { 2. ¿Cómo se acumula y se } \\
\text { registra el costo en la } \\
\text { empresa? } \\
\text { 3. ¿Cuáles son los } \\
\text { métodos de registro y } \\
\text { acumulación del costo en } \\
\text { la entidad? }\end{array}$ & Contabilidad \\
\hline $\begin{array}{l}\text { Sistema } \\
\text { Financiero }\end{array}$ & $\begin{array}{l}2 . \quad \text { Identificar las } \\
\text { principales } \\
\text { instituciones de } \\
\text { carácter bancario } \\
\text { existentes en un país } \\
\text { en el mundo actual. } \\
3 . \quad \text { Identificar los } \\
\text { principales Activos } \\
\text { Financieros a Largo y } \\
\text { a Corto Plazo y } \\
\text { aprender sus } \\
\text { características } \\
\text { importancia para el } \\
\text { funcionamiento } \\
\text { financiero eficaz de la } \\
\text { empresa. }\end{array}$ & $\begin{array}{l}\text { 4. ¿Con qué tipo de } \\
\text { institución financiera de } \\
\text { carácter bancario la } \\
\text { empresa tiene establecido } \\
\text { contratos? } \\
\text { a) Diga su función. } \\
\text { 5. Mencione los Activos } \\
\text { Financieros a Corto y } \\
\text { Largo Plazo de la empresa } \\
\text { y comentar sus } \\
\text { características y formas de } \\
\text { registro posible. }\end{array}$ & Finanzas \\
\hline $\begin{array}{l}\text { Contabilidad } \\
\text { General IV }\end{array}$ & 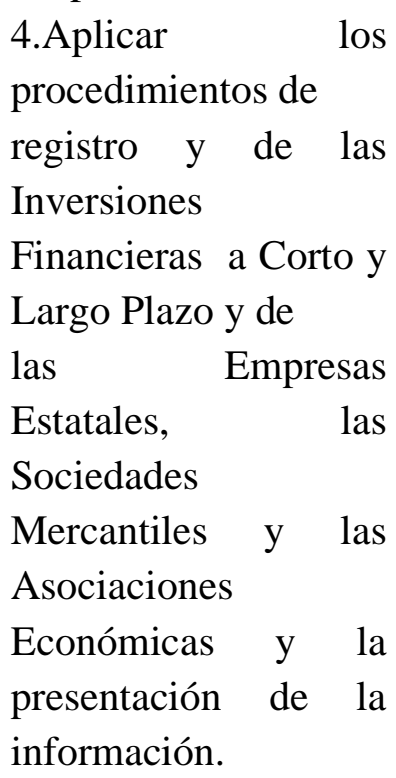 & $\begin{array}{l}\text { 6. Verifique en la empresa } \\
\text { la presencia de préstamos } \\
\text { a corto y largo plazo. } \\
\text { a) Identifique los tipos de } \\
\text { hechos económicos. } \\
\text { b) Registre los hechos } \\
\text { económicos relacionados } \\
\text { con los compromisos de } \\
\text { pago. } \\
7 . \text { Registre los hechos } \\
\text { económicos vinculados } \\
\text { con las Inversiones } \\
\text { Financieras a Corto Plazo, } \\
\text { a Largo Plazo y el Capital. }\end{array}$ & Contabilidad \\
\hline
\end{tabular}




\begin{tabular}{|c|c|c|c|}
\hline la Gestión & $\begin{array}{l}\text { 5.Identificar } \\
\text { ventajas del sistema } \\
\text { operativo como un } \\
\text { entorno de trabajo } \\
\text { Gráfico y sus } \\
\text { comandos } \\
\text { significativos. }\end{array}$ & $\begin{array}{l}8 . \text { Explique las } \\
\text { características del } \\
\text { software contable que se } \\
\text { utiliza en entidad en } \\
\text { cuanto a sus ventajas y } \\
\text { limitaciones, estructura } \\
\text { modular. } \\
\text { 9. Realice capturas de } \\
\text { pantalla con algunos } \\
\text { comprobantes del registro } \\
\text { de los hechos } \\
\text { económicos. }\end{array}$ & \\
\hline $\begin{array}{l}\text { Contabilidad } \\
\text { General III }\end{array}$ & $\begin{array}{l}\text { 6.Aplicar técnicas de } \\
\text { valuación, registro y } \\
\text { presentación de las } \\
\text { partidas } \\
\text { fundamentales que } \\
\text { integran el activo fijo, } \\
\text { el Estado de Situación } \\
\text { así como las fuentes } \\
\text { de financiamiento a } \\
\text { corto y largo plazo, } \\
\text { teniendo siempre en } \\
\text { cuenta el } \\
\text { cumplimiento del } \\
\text { marco conceptual de } \\
\text { la Contabilidad. }\end{array}$ & $\begin{array}{l}\text { 10. Analice la } \\
\text { composición de las } \\
\text { partidas incluidas en el } \\
\text { Estado de Situación. } \\
\text { 11.Fundamente } \\
\text { teóricamente la } \\
\text { Evaluación y presentación } \\
\text { de las partidas en los } \\
\text { Estados financieros } \\
\text { referente a los Activos } \\
\text { Fijos. las } \\
\text { 12. Analice } \\
\text { legislaciones vigentes en } \\
\text { la actividad económica y } \\
\text { contable de la empresa } \\
\text { relacionado con la } \\
\text { nómina, maternidad, el } \\
\text { subsidio a los } \\
\text { trabajadores. Su } \\
\text { procedimiento contable y } \\
\text { la presentación de las } \\
\text { partidas asociadas en el } \\
\text { Estado de Situación. } \\
\text { 13. Registre los hechos } \\
\text { económicos relacionados } \\
\text { con los Activos Fijos y } \\
\text { Pasivos Corrientes y a } \\
\text { Largo Plazo. }\end{array}$ & Contabilidad \\
\hline Finanzas & $\begin{array}{l}\text { 7. Valorar } \\
\text { importancia }\end{array}$ & $\begin{array}{l}\text { 14. Verifique si fuera una } \\
\text { empresa presupuestada el }\end{array}$ & Estudiante \\
\hline
\end{tabular}




\begin{tabular}{llrl}
\hline Gubernamental & sistema & & papel que desempeña la \\
es & presupuestario & y la & tesorería en Cuba y la \\
& estructura & del & estructura del presupuesto \\
& presupuesto & del & del Estado en Cuba. \\
& Estado. & &
\end{tabular}

Teoría

Sociopolítica

Estadística

Matemática

Econometría

8. Valorar desde una
perspectiva
académica,
marxista

tercermundista las principales teorías y núcleos conceptuales que se aplican al análisis de los procesos políticos más puntuales y, en particular, el proyecto político de la Revolución Cubana.

9. Obtener una muestra aleatoria de los comprobantes de los hechos económicos de la entidad.

10. Obtener un modelo de regresión que permita establecer la relación entre las variables con que trabaja la empresa.
15. Mencione las leyes, Departament reglamentos y políticas o establecidas que se rige la Jurídico y entidad.

16. Mencione los procedimientos contables empleados en la empresa. 17. Argumente a cuáles de los

Lineamientos de la política económica la empresa le brinda una mayor atención.

18. Escoja una muestra de Contabilidad los comprobantes del registro de los hechos económicos de la empresa para conocer las cuentas usadas, los tipos de hechos económicos, lo documentos que avalan las operaciones contables.

19. ¿Cuáles son las Finanzas variables más significativas con que trabaja la empresa para su correcto funcionamiento? a) ¿Cuál es la variable dependiente y cuál es la independiente?

b) Determine las variables

dependientes cuales influyen

sobre la dependiente. 
Otras preguntas necesarias:

$\begin{array}{lll}\text { Contabilidad } & \text { 12. Constatar aspectos } & 20 . \quad \text { Solicite algunos Contabilidad } \\ \text { relacionados con el } & \text { documentos mercantiles y } \\ \text { registro. } & \text { describa como se } \\ & \text { fiscalizan en la empresa. } \\ & \text { ¿Qué normas se ponen en } \\ & \text { práctica o postulados de la } \\ \text { información financiera? } & \text { 20. Fotografíe cada } \\ & \text { documento que ampara el } \\ & \text { registro. Ejemplo: factura } \\ & 21 . \text { Explique cómo se } \\ \text { identifican las cuentas y } & \text { subcuentas. }\end{array}$

c) Compruebe los

supuestos

básicos del modelo de

regresión.

Estructura del informe:

1. Portada: debe incluir nombre de la universidad, facultad, departamento.

2. Resumen (máximo 250 palabras, en idioma inglés y español. Debe contener objetivos del trabajo y principales resultado obtenidos en función de la entidad).

1. 3.Índice

3. Introducción: con la caracterización de la empresa y breve fundamentación teórica sobre la importancia de la Contabilidad, los registros y tipos de hechos económicos, normativa y procedimientos contables y los estados financieros así como las cuentas desde la perspectiva de las definiciones estudiadas.

4. Desarrollo: incluye las tareas específicas a desarrollar.

5. Conclusiones: Utilidad de los conocimientos adquiridos a través del desarrollo de la Práctica Laboral Contable y Financiera I.

6. Recomendaciones: Sugerencias que contribuyan a incrementar la calidad del desarrollo de la práctica laboral.

7. Bibliografía: Normas Generales de Contabilidad, documentación revisada de la empresa, documentos elaborados por los profesores de las asignaturas, bibliografía de las asignaturas.

8. Anexos: Clasificador de cuentas, modelos que utilizan, organigrama, estructura organizativa, documentos mercantiles. Resoluciones vigentes y aval del tutor donde se tiene en cuenta la asistencia, puntualidad, relaciones interpersonales, motivación, desempeño demostrado en el área contable, disposición, compromiso y responsabilidad en las tareas encomendadas, logros y limitaciones en la práctica con énfasis en el registro de los hechos económicos. 
Nota: el informe debe estar redactado con letra Arial 12, interlineado 1.5, se debe entregar en formato digital, papel y realizar una presentación de power point que debe contener al menos portada, introducción, desarrollo, conclusiones y recomendaciones.

Asignaturas del año:

\begin{tabular}{ll}
\hline Asignaturas del semestre I & Asignaturas del semestre II \\
\hline Contabilidad General III & Econometría \\
Estadística Matemática & Investigación de Operaciones \\
Sistema Financiero & Contabilidad General IV \\
Teoría Sociopolítica & Finanzas Gubernamentales \\
Elementos de Pedagogía & Contabilidad de Gestión I \\
Informática para la gestión & Practica Laboral Contable y Financiera I \\
Inglés II & Inglés III \\
Educación Física III & Educación Física IV \\
\hline
\end{tabular}

\section{Resultados}

Con la aplicación de la guía de la Práctica Laboral Contable y Financiera I, el 100\% de los estudiantes presentaron un aval de la entidad donde realizaron las prácticas donde se evidencian sus desempeños en las empresas y un anexo al informe de la práctica con un ejercicio del registro de los hechos económicos de los Activos y Pasivos Corrientes, a Largo Plazo, Capital, los Ingresos y Gastos así como los documentos mercantiles, los tipos de hechos económicos más utilizados, sustentados en los procedimientos contables, para ello, se apoyaron en los conocimientos de las asignaturas Contabilidad General I, II, III y IV y Contabilidad de Gestión I.

El $40 \%$ de los estudiantes, dieron criterios positivos del trabajo de los compañeros, aunque el 30.76\% lograron señalar aspectos que debían ser profundizados como el registro más detallado de los hechos económicos, porque en los casos presentados solo se tuvieron en consideración las cuentas y el $29.23 \%$ no emitieron ningún criterio.

En su totalidad, los estudiantes, profesores y tutores se manifestaron satisfechos con la introducción de tareas donde se cumplimentan los objetivos generales de las asignaturas que integran el año, en concordancia con las tareas específicas de los puestos de trabajo del departamento económico de las empresas del territorio, la definición de la estructura del informe de la práctica y el seguimiento y control de las actividades tanto en la solicitud y constatación de la información necesaria para dar cumplimiento a la guía como en las funciones otorgadas en los puestos de trabajo como parte de su entrenamiento.

\section{Conclusiones}

- La propuesta de la guía de la Práctica Laboral Contable y Financiera I, contribuyó en la sistematización de los contenidos de las asignaturas del año, con la inserción de tareas para cada una de las asignaturas, objetivos y la ubicación que se ejemplificaron sus resultados a través de un informe final, acompañado de un aval 
de la empresa y un ejercicio anexado con el registro de los principales hechos económicos, los documentos mercantiles, procedimientos contables, las cuentas y características de la organización.

- Se realizó un intercambio entre profesores del colectivo de segundo año y los estudiantes así como los tutores de las empresas a través de la inserción de los estudiantes en las tareas de acompañamiento de conjunto con los especialistas de gestión económico financiera de las empresas, con énfasis en las tareas relacionadas con el registro de los hechos económicos.

- Los resultados de los estudiantes evidencian conocimientos, habilidades, desempeños satisfacción y valores éticos del contador, durante el proceso de las prácticas así como la vinculación del estudio con el trabajo.

\section{Referencias bibliográficas}

Hernández, G. C. (2014). Las competencias profesionales y la gestión de su mejora en procesos contables. Acercamiento teórico (CD-ROM). Matanzas. Universidad de Matanzas Camilo Cienfuegos.

MES. (2017). Plan de Estudio E. La Habana (Cuba).

Prado, E, y Baujín, P. (2020). La formación de profesionales en las Ciencias Contables y Financieras, con énfasis en el registro de los hechos económicos y su relación con las etapas de la economía cubana. X 1. En Arboleda, C (Presidencia). IX Congreso Internacional de Educación y Pedagogía.

Prado, E. (2017). Hiperambiente de aprendizaje para a formaçäo da competencia registar factos econòmicos. En J.N, Silvestre (Presidencia), IV Jornada Científico Pedagógica da Faculdade de Economía. Jornada dirigida pela Universidade Katyavala Bwila Benguela.

Prado, E. (2018a). Procedimento para a formaçäo da competencia registar factos económicos asociados ao Inventario. En M.J, da Costa (Presidencia), IV Jornada Científica Pedagógica. Jornada dirigida pelo Instituto Superior da Ciencias da Educaçäo. Huambo.

Prado, E. (2018b). Estrategia didáctica para registar factos económicos na carrera Contabilidade VII. En I.M, Romero (Presidencia), Jornada Científico Pedagógica da Región Académica II Benguela-Kuanza-Sul. Jornada dirigida pela Universidade Katyavala Bwila. Kuanza -Sul.

Prado, E., (2021). La formación inicial de la competencia profesional específica registrar hechos económicos mediante la Contabilidad General en la carrera Contabilidad y Finanzas. (Tesis doctoral en Ciencias Pedagógicas, Universidad de Ciencias Pedagógicas Enrique José Varona. La Habana, Cuba).

Prado, E., González, M., y Baujín, P. (2021). La formación inicial de la competencia profesional específica registrar hechos económicos mediante la Contabilidad General en la carrera 
Contabilidad y Finanzas..Editorial Académica Española. España. ISBN 978-620-303824-8. Disponible en: httpp/ www.eae.publishing com.

Prado, E., González, M., y Montalvo, I. (2021). La práctica laboral y su incidencia en la formación inicial de la competencia registrar hechos económicos. En B, Edgar (Presidencia), X Convención Científica Internacional de la Universidad de Matanzas.

Prado, E., y López, L. (2020). El registro de los hechos económicos en la actividad comercial, como punto de partida en la enseñanza de la Auditoría. Revista de Auditoría y Control, 40(1), p. 94-100. Recuperado de: http//contraloría.gob.cu.

Valera, L. (2009). Propuesta de competencias profesionales para los estudiantes de la carrera de Contabilidad y Finanzas. Cuaderno de Educación y Desarrollo 1(1), sp. Recuperado de: http://scielo.sld.cu/scielo.php.

Valera, L. (2016). Modelo Pedagógico de la formación de competencias profesionales en la disciplina principal integradora de la carrera Contabilidad y Finanzas Didáctica y Educación. Revista de Educación Superior, 2(4), sp. Recuperado de: http://scielo.sld.cu/scielo.php. 


\section{Para citar el artículo indexado}

Prado Chaviano, E., González González, M., Jiménez Valero, B., Montalvo Palacios, I., \& Velastegui López, L. E. (2021). La práctica laboral y su incidencia en la formación inicial de la competencia registrar hechos económicos. AlfaPublicaciones, 3(2.1), 100112. https://doi.org/10.33262/ap.v3i2.1.52

\section{Liencia}

El artículo que se publica es de exclusiva responsabilidad de los autores y no necesariamente reflejan el pensamiento de la Revista Alpha Publicaciones.

El artículo queda en propiedad de la revista y, por tanto, su publicación parcial y/o total en otro medio tiene que ser autorizado por el director de la Revista Alpha Publicaciones.
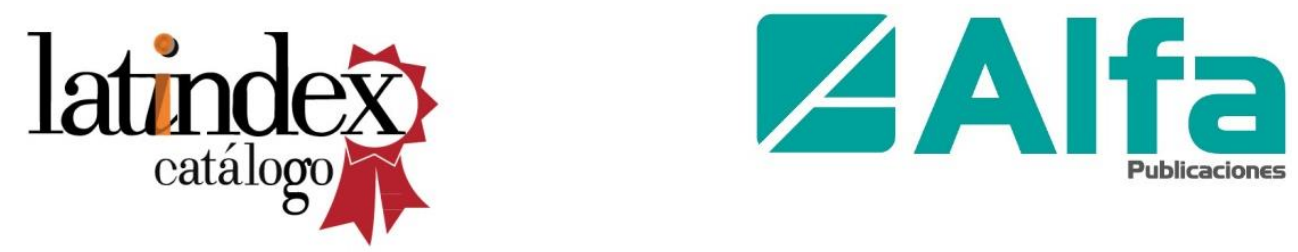